\title{
MUSCLE PERFORMANCE, BODY FAT, PAIN AND FUNCTION IN THE ELDERLY WITH ARTHRITIS
}

\author{
Wagner Teixeira dos Santos ${ }^{1}$, ERIKa de Carvalho Rodrigues ${ }^{1}$, Míriam Raquel Meira Mainenti ${ }^{1}$
}

\section{ABSTRACT}

Objective: To correlate muscule performance, body composition, pain and joint function in elderly people with gonarthrosis. Method: 21 elderly patients were submitted to bioelectrical impedance analysis, dynamometry associated with electromyographic (EMG) evaluation of isometric knee extension, in addition to pain assessment by the Numeric Pain Intensity Scale and function assessment, by the Western Ontario and McMaster Universities (WOMAC) Osteoarthritis (OA) questionnaire. Correlations were checked by the Pearson's correlation coefficient. Results: The sample characteristics were mean age $67.36 \pm 4.21$ years old, body fat percentage $40.57 \pm 6.15 \%$, total WOMAC score $43.27 \pm 16.32 \%$, and maximum strength $19.95 \pm 6.99 \mathrm{kgF}$. Pain during movement showed a statistical association with WOMAC physical activity domain $(r=0.47)$ and its general score $(r=0.51)$; pain intensity at night presented association with WOMAC stiffness domain $(r=0.55)$, in addition to the negative correlation with the slope values of the Medium Frequency of the EMG signal $(r=-0.57)$. Conclusion: pain intensity is correlated to functional incapacity in elderly people with knee OA and to a greater expression of fatigue in EMG signal. Levels of Evidence III, Study of non consecutive patients

Keywords: Osteoarthritis. Pain. Muscle strength. Knee. Aged.

Citation: Santos WT, Rodrigues EC, Mainenti MRM. Muscle performance, body fat, pain and function in older adults with arthritis. Acta Ortop Bras. [online]. 2014;22(1):54-8. Available from URL: http://www.scielo.br/aob.

\section{INTRODUCTION}

In recent decades, there has been a significant increase in life expectancy of people in developed and underdeveloped countries. Kurek et al. ${ }^{1}$ found that the number of elderly people increases in a greater proportion in relation to people who are born, making the aging process be considered an important issue by the government and the society in many countries. As men age, their overall health state undergoes several transformations, including an increased risk of developing chronic diseases (hypertension, diabetes mellitus, heart diseases), musculoskeletal dysfunctions, decline in sexual potency, decline in organic capacity, among others. ${ }^{2}$

Among the diseases that affect joint function in the elderly, osteoarthritis $(\mathrm{OA})$, degenerative disease also known as osteoarthritis, is the one that most affects the locomotor system. ${ }^{3}$ It is estimated that $4 \%$ of the Brazilian elderly population has OA, being the knee joint the second most affected by the disease, with $37 \%$ of cases ${ }^{4}$ Knee OA presents as clinical symptoms: bone deformities, pain, stiffness, decrease or loss of joint function, decreased muscle mass, strength and resistance, consequently leading to limitations in daily life activities. ${ }^{3}$

Among the clinical characteristics mentioned above, the de- crease in strength and occurrence of early muscle fatigue, especially of the knee extensor muscles, is the second most frequent complaint reported by older adults with a diagnosis of gonarthrosis, only behind pain complaint. ${ }^{3}$ However, the existence of a relationship between these symptoms is not yet fully established.

A recent study correlated the performance of flexors and extensors knee muscles on an isokinetic dynamometer and the pain, stiffness and function domains of the Western Ontario McMaster Universities Osteoarthritis Index Questionnaire (WO$\mathrm{MAC}$ ) in a cross-sectional study in elderly women with OA knee. The study concluded that lower muscle strength and resistance and the presence of imbalance of the knee muscles correlated negatively with all WOMAC domains. ${ }^{5}$ However, there were no other studies to corroborate this finding, even when assessing other types of muscle contraction, as isotonic or isometric, which are most commonly used in daily life activities. Moreover, it is necessary to know the levels of muscular strength and resistance, as well as quantifying the symptom pain (both at movement and at rest) and functionality in patients with $\mathrm{OA}$. There are few studies that present these data and correlate

All the authors declare that there is no potential conflict of interest referring to this article.

1. Centro Universitário Augusto Motta (UNISUAM), Bonsucesso, RJ, Brazil.

Research performed at the Laboratory of Human Movement Analysis, Centro Universitário Augusto Motta, Bonsucesso, RJ, Brazil.

Correspondence: Míriam Raquel Meira Mainenti. Programa de Pós-Graduação em Ciências da Reabilitação. Praça das Nações, 34, 3o andar, Bonsucesso, RJ, Brazil. $21041-021$. miriam.mainenti@hotmail.com 
muscle behavior with subjective variables of pain and function. Thus, this study aimed to quantify the isometric muscle performance, body composition, levels of pain and function in older adults with knee OA, besides verifying the existence of association between objective strength measurements and muscle fatigue with the subjective variables of pain and joint function.

\section{MATERIALS AND METHODS}

This is a cross-sectional study, conducted at the Laboratory of Human Movement Analysis at Centro Universitário Augusto Motta (UNISUAM, RJ) in elderly patients diagnosed with unilateral or bilateral gonarthrosis. The recruitment of volunteers for this research was conducted at the screening session of the Centro Municipal de Reabilitação do Engenho de Dentro, RJ, by verbal communication invitations to participate in research, between July and October 2012. The protocol was approved by the Ethics Committee of Institutional Research (CAAE: 03105612.5.0000.5235) and all patients signed an informed consent before joining the study. All evaluations were performed on the same day. The patient underwent a physical examination to obtain information on its clinical and general condition. Range of motion for knee flexion and extension (manual goniometer CARCI, São Paulo, Brazil) and the degree of manual muscle strength of the compromised lower limb using the criteria of Kendall et al. ${ }^{6}$ were measured. In cases of bilateral OA, we analyzed the knee that had the higher pain intensity. Then the functional WOMAC questionnaire was applied and the following measurements were performed: anthropometry, total and segmental bioelectrical impedance of the lower limbs, strength and resistance to muscle fatigue, as it will be detailed further.

\section{Participants}

Participated in the study seniors who met the following inclusion criteria: age $\geq 60$ years; literate of both genders; released by medical staff for physical therapy, with a confirmed medical diagnosis of uni- and/or bilateral knee osteoarthritis level one and two according to clinical and radiographic criteria of the American College of Rheumatology, ${ }^{3}$ range of motion for knee flexion of at least $90^{\circ}$ and knee extension with a maximum reduction of $10^{\circ}$, confirmed by goniometric evaluation (CARCl, São Paulo, Brazil), strength of quadriceps and ischiotibials from grade three in the classification of hand force table; 6 independent ambulation; intensity of referred pain from zero to four in

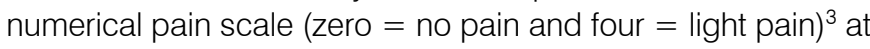
the day of assessment; who agreed to participate voluntarily in the study and who had not participated in any building muscle program for in the last six months. Volunteers, who had hypertension, and/or uncontrolled heart disease, diabetes mellitus, neurological diseases, used a pacemaker, or dysfunctions in other joints of the body that could hamper the achievement of physical exercise, were excluded from the study.

\section{Anthropometric measurements}

Anthropometric measurements included total body mass (TBM; kg), height $(\mathrm{m})$ and lower limb length. In order to measure the height and MCT analog scale $(0.1 \mathrm{~kg})$ with a coupled $(0,005 \mathrm{~m})$ stadiometer (R110, Welmy, Santa Bárbara do Oeste, São Paulo, Brazil) was used. To check the length of the lower limbs, a measurement tape (Terrazul, Cambuci, São Paulo, Brazil) was used, positioned Acta Ortop Bras. 2014;22(1):54-8 between the anatomical points anterior superior iliac spine and lower edge of the malleolus tibial. ${ }^{7}$

\section{Body composition}

To assess the body composition the tetrapolar bioelectrical impedance method was used through the BIA 310e bioimpedance analyzer (Biodynamics - Seattle, Washington, USA), which produces an alternating electric current at a frequency of $50 \mathrm{kHz}$, imperceptible to humans. This analysis was performed on two approaches: the total body and segmental limb. For total body bioimpedance, electrodes were placed in regions of the dorsum of the right hand and foot ${ }^{8}$ and for segment evaluation the same electrodes were placed bilaterally in regions of the anterior superior iliac spine and medial malleolus. ${ }^{7}$ In order to measure fat-free mass, the equation of Kyle et al. ${ }^{8}$ was used: $-4.104+0.518 . E^{2} / R$ $+0.231 \times \mathrm{TBM}+0.130 . \mathrm{Xc}+4.229$.sex, where $\mathrm{H}=$ height $(\mathrm{cm})$, $\mathrm{R}=$ resistance $(\Omega), \mathrm{TBM}=$ total body mass $(\mathrm{kg}), \mathrm{Xc}=$ reactance $(\Omega)$ and sex $=0$ for women and 1 for men. For the estimation of lean mass segment, the ratio of the square of the length and strength of the lower limb (lenghth²/R) which, in a previous study, correlated with the local lean mass. ${ }^{?}$

\section{Strength and Muscle Fatigue}

The test of strength and resistance to muscle fatigue was associated with the use of three features: fitness station EMK 1500 (Kenkorp, São Paulo, Brazil), a EMG -810 surface electromyograph (EMG system of Brazil LTDA , São Paulo , Brazil), and a DIN_TRO traction dynamometer (EMG System do Brazil LTDA, São Paulo, Brazil). For the strength test, the patient was asked to perform, with the knee at $90^{\circ}$, three maximal isometric knee extensions for five seconds with 30 seconds rest between each attempt. The maximum value reached (MVIC - maximal voluntary isometric contraction) was chosen, and from this result, it has been selected $50 \%$ of this burden as a target for the reported conduct an isometric contraction of the knee for one minute. To assist the patient in achieving the assessed level of force required, a visual monitoring unit showing a blue band with the plus and minus margin of $10 \%$ of the calculated value was made through a computer monitor. The analysis of muscle fatigue was performed by surface electromyography (EMG). The electrodes were placed over the medial vastus muscle of the lower limb that had a greater commitment by osteoarthritis following the recommendations of the SENIAM group. ${ }^{9}$ The distance between the electrodes was $20 \mathrm{~mm}$, and the reference electrode was placed over the contralateral medial malleolus. Active differential electrodes (20x gain) were used to collect EMG signals from surface electrodes for the $A / D$ converter. The sampling frequency was $1000 \mathrm{~Hz}$ and the signals were amplified with a 2000x gain. We applied a Butterworth bandpass filter ( $2^{\text {nd }}$ order, bidirectional) frequency band between 5 and $450 \mathrm{~Hz}$. The analysis of the collected signal during one minute isometric contraction was performed immediately after. The calculated parameters were: Root Mean Square, RMS), related to the number of active motor units (signal amplitude) and median frequency (MF), related to the shots of the action potential of fiber contraction. Using linear regression lines were fitted to the RMS and MF values throughout the isometric contraction plateau (60 seconds). The angular coefficients of the fitted lines were named slope RMS and slope MF, respectively. The 
signals were stored and analyzed by the SuiteMYO software (PhD² Consultoria e Sistemas Ltda, RJ, Brazil). After completion of the protocol, the level of perceived exertion using the Borg scale was evaluated.

\section{Functionality of Lower Limbs}

In this study the WOMAC questionnaire (Western Ontario and McMaster Universities Osteoarthritis Index), validated for Portuguese (Brazil) language by Fernandes was used. ${ }^{10}$ This instrument was employed to assess pain, joint stiffness and physical activity in patients with osteoarthritis of the knee and hip, consisting of 24 questions. The areas receive points ranging from 0 to 20 (pain), 0 to 8 (stiffness) and 0 to 68 (physical activity). For the analysis it was verified the percentage score for each domain and for the total instrument. ${ }^{5,10}$ The higher the score, the worse the evaluation of the participant. Also a numerical scale was used, ranging from 0 to 10 , to assess pain intensity. The volunteer was questioned on pain during daily movements at rest and at night, with a focus in the last 72 hours, and the perception of pain at the time of evaluation.

\section{Statistical Analysis}

Data distribution was checked by the Kolmogorov-Smirnov test. As the main variables were normally distributed, parametric approaches for analyzes were selected. Data were presented as mean \pm standard deviation, and the association between variables was represented by the Pearson correlation coefficient (r), associated with the value of p. All analyzes and graphs were performed using the software SPSS (version 13.0), considering significant the analyzes with $p$-values $\leq 0.05$. It was defined as a strong positive correlation $p$ values between 0.70 to 1.0; moderate, 0.30 to 0.70 ; weak between 0 and 0.30 ; and a strong negative correlation $p$ values between -0.70 to -1 ; moderate, 0.30 to -0.70 ; and weak 0 to $-0,30 .{ }^{11}$

A sample of 20 volunteers was estimated for a minimal association with the 0.54 significance level $(\alpha)$ of $5 \%$ and power $(\beta)$ of $80 \%$. The sample size was estimated from an algebraic implementation that calculates the minimum correlation coefficient to reject the null hypothesis in each sample size. ${ }^{12}$

\section{RESULTS}

The study sample consisted of 21 elderly patients, 18 of them were women, mean age $67.36 \pm 4.21$ years old. (Table 1) The subjects' body composition and muscle performance in maximal strength and fatigue resistance test are shown in Table 2. The pain, stiffness and physical activity WOMAC domains showed average values of $43.40 \pm 16.50,45.45 \pm 26.03$ and $42.90 \pm 18.02 \%$, respectively. The overall average score was $43.27 \pm 16.32 \%$. Regarding the numerical pain scale, patients reported an average value of $5.57 \pm 2.60$ for pain on movement, $2.81 \pm 2.52$ for nocturnal pain, and $1.66 \pm 2.78$ for pain at rest. Moderate associations were found for the numerical scale for pain motion and physical activity domain, and overall WOMAC score. (Table 3) The values assigned to the numerical scale of nocturnal pain showed moderate correlation with the WOMAC domain stiffness, in addition to negative correlation with slope values of median frequency, associated with fatigue. (Table 3) Another significant result was the identification of moderate correlations between age and the WOMAC domain pain $(r=0.46, p=0.03)$, and WOMAC total score $(r=0.41, p=0.05)$. In addition to these associations, a moderate correlation between lean mass and MVIC $(p=0.04 r=0.48)$ was observed.

Regarding total WOMAC score, weak correlations were found with the MVIC values, the RMS Slope and MF Slope. (Table 3)

\begin{tabular}{c|c}
\multicolumn{2}{l}{ Table 1. Characteristics of the sample. } \\
\hline Variable & Mean \pm St. Dev. \\
\hline Total Body Mass (kg) & $75.19 \pm 14.67$ \\
\hline Right Knee* $(\%)$ & 61.90 \\
\hline Bilateral compromise (\%) & 80.99 \\
\hline Height (m) & $1.52 \pm 0.57$ \\
\hline BMl (kg/m²) & $32.36 \pm 5.98$ \\
\hline Time with osteoarthrosis (months) & $101.68 \pm 121.25$ \\
\hline AOM of knee flexion (degrees) & $113.55 \pm 12.13$ \\
\hline AOM of knee extension (degrees) & $171.95 \pm 6.70$ \\
\hline
\end{tabular}

St.Dev: Standard deviation; BMI: Body Mass index; AoM: Arc of Motion; ${ }^{*}$ Prevalence of unilateral right arthrosis + bilateral with concentrated pain in the right limb.

Table 2. Body composition and muscle performance of the participants

\begin{tabular}{|c|c|}
\hline Variable & Mean \pm St. Dev. \\
\hline Lean mass $(\mathrm{kg})$ & $43.02 \pm 8.16$ \\
\hline Fat mass $(\mathrm{kg})$ & $29.64 \pm 7.45$ \\
\hline Relative fat (\%) & $40.57 \pm 6.15$ \\
\hline Length2/ResR $\left(\mathrm{cm}^{2} / \Omega\right)$ & $27.42 \pm 4.08$ \\
\hline Length2/ResL $\left(\mathrm{cm}^{2} / \Omega\right)$ & $27.03 \pm 4.69$ \\
\hline Slope RMS & $0.631 \pm .558$ \\
\hline Slope MF & $-0.446 \pm .476$ \\
\hline MVIC (kgF) & $19.95 \pm 6.98$ \\
\hline
\end{tabular}

St. Dev: Standard deviation; Length2/ResR: Ratio between square length and the resistance of the right lower limb; Length2/ResL: Ratio between square length and the resistance of the left lower limb; RMS: Root mean square; MF: Median Frequency; MVIC : Maximum voluntary isometric contraction.

\section{DISCUSSION}

The patients studied presented mean arc of motion for knee flexion $\left(113.55^{\circ}\right)$ and extension $\left(171.95^{\circ}\right)$ below the reference values cited by Charro et al..$^{13}\left(140^{\circ}\right.$ and $180^{\circ}$, respectively). The mean BMl found $\left(32.36 \mathrm{~kg} / \mathrm{m}^{2}\right)$ classifies the sample as Obesity grade $1 .{ }^{14}$ The average degree of relative fat of the sample is smaller than the median found by Mainenti et al. ${ }^{15}$ in a sample of physically active elderly people (40.6 vs. 44.3), but still considered an obese sample, according to the classification adopted by Deurenberg et $\mathrm{al} .{ }^{16}>35 \%$ for women and $>25 \%$ for men, regardless the age.

The average value of the MVIC for the knee extension movement 
Table 3. Pearson's correlation for the analysis performed.

\begin{tabular}{|c|c|}
\hline Variables & $r$ (Pearson's) \\
\hline PNS movement and stiffness WOMAC & 0,34 \\
\hline PNS movement and PA WOMAC* & 0,47 \\
\hline PNS movement and WOMAC overall* & 0,51 \\
\hline PNS movement and CIVM & 0,01 \\
\hline PNS movement and RMS slope* & $-0,54$ \\
\hline PNS movement and MF slope & 0,26 \\
\hline PNS night time and stiffness WOMAC* & 0,56 \\
\hline PNS night time and MF WOMAC & 0,14 \\
\hline PNS night time and WOMAC overall & 0,27 \\
\hline PNS night time and MVIC & $-0,06$ \\
\hline PNS night time and RMS slope & 0,31 \\
\hline PNS night time and MF*slope & $-0,57$ \\
\hline Stiffness WOMAC and MVIC & $-0,04$ \\
\hline Stiffness WOMAC AND slope RMS & 0,13 \\
\hline Stiffness WOMAC and MF slope & $-0,19$ \\
\hline PA WOMAC AND MVIC & 0,16 \\
\hline PA WOMAC and RMS slope & 0,02 \\
\hline PA WOMAC and MF slope & 0,10 \\
\hline WOMAC overall and MVIC & 0,13 \\
\hline WOMAC overall and RMS slope & $-0,01$ \\
\hline WOMAC overall and MF slope & 0,09 \\
\hline
\end{tabular}

${ }^{*} p \leq 0,05$; PNS: Pain numeric scale; WOMAC: Western Ontario and McMaster Universities Osteoarthritis Questionnaire; PA: Physical Activity; MVIC: Maximum voluntary isometric contraction; RMS: arthritis Questionnaire; PA: Physical Activity;

found in this study (19,95 kgF) is close to those found by Becker et $\mathrm{al} .{ }^{17}$ in non-operated knees of patients with osteoarthritis $(14,52 \mathrm{kgF})$, but substantially higher than those observed by Pap et al. ${ }^{18}$ in patients with osteoarthritis symptoms for over 60 months $(7.84 \mathrm{kgF})$. The average slope value for RMS (0.631) and MF (-0.446) staked out a more intense process of fatigue than the one presented by Gonçalves and Silva ${ }^{19}(0.21$ and -0.09 , respectively), in a sample of young adults (mean age 19.7 years old). The authors did not find papers that performed this same kind of analysis in the elderly. Regarding the domains of the WOMAC questionnaire, the average values of this study (43.27\%) were similar to those found by Fernandes ${ }^{10}(41.54 \%)$ in individuals aged $40-80$ years old, and by Santos et al. ${ }^{5}$ (46.64\%), in patients with osteoarthritis of the lower limbs above 65 years of age.

Foley et al. ${ }^{20}$ conducted a cross-sectional study of elderly men and women, mean age $62.5 \pm 7.4$ years. To evaluate the risk of falls in elderly a short review of the physiological profile (Prince of Wales Medical Research Institute) was used. The results in this study showed a significant association between pain and functional capacity as predictive risk factor for falls in the elderly. The fact that the data show no association between muscle performance and total WOMAC score can be explained based Acta Ortop Bras. 2014;22(1):54-8 on the type of contraction frequently used in daily life of the elderly. There is a much greater use of isotonic rather than isometric contractions, which was evaluated in this study. However, the small sample size may have also influenced this analysis. Santos et al..$^{5}$ evaluated 80 elderly patients (71.2 \pm 5.3 years) with clinical diagnosis of knee $\mathrm{OA}$ and found an inverse correlation between all domains of the WOMAC and isokinetic muscle strength of knee extensors and flexors, suggesting that the individuals evaluated had reduced muscle strength and a negative impact in the areas of pain, stiffness and mainly joint function. A moderate negative correlation between nocturnal pain and slope of the median frequency of muscle EMG activity $(r=-0.57)$ in the present study highlights that pain can influence the inhibition of efferent firings to the muscle fibers, since it is known that the median frequency is related to the firing rate of action potential in the muscle and the rate of reduction of shots (with consequent reduction in the median frequency) is associated with fatigue. ${ }^{21}$ The coefficient found presents sufficient value ${ }^{12}$ to reject the null hypothesis of no correlation, considering the 21 participants and the standards of reliability and power previously mentioned in this study. For this sample size, the minimum coefficient would be $0.53 .^{12}$

The musculoskeletal system is one of the most affected by aging, especially with the development of degenerative diseases that compromise their functional capacity, and hence the quality of life. This statement explains the correlation found in this study between age, pain and general domain of the WOMAC score.

By correlating lean mass and maximal voluntary isometric contraction it has been observed that the higher the lean mass, the better muscle performance, corroborating the study by Orsatti et al., ${ }^{22}$ who found in 52 sedentary women, aged between 40 and 70 years, the same relationship between lean body mass and muscle strength. Carmeli et al. ${ }^{23}$ showed a strong correlation between the status pre-sarcopenia and sarcopenia. They also claim that the change in quadriceps muscle strength is a measure related to muscle age and it is strongly associated with physical and functional decline.

The present study has some limitations, such as small sample size and the performance only of isometric contractions in assessing muscle performance. However, the analysis of muscle fatigue by the behavior of the root mean square and median frequency of the myoelectric signal in the elderly is still quite scarce in the literature, highlighting the findings of this study and encouraging further research in this area.

\section{CONCLUSION}

Mean values of the sample point to a group with high body fat, high expression of fatigue and functionality of lower limbs similar to published studies. The objective measurements of muscle strength and fatigue were not associated with subjective variables of joint function (WOMAC), but with those related to pain in patients with osteoarthritis of the knee. Moreover, the values assigned to this scale and some domains of the WOMAC also correlated positively. Pain intensity is correlated with functional disability of older individuals with knee OA and a more significant event of signs of fatigue, checked the behavior of the median frequency of the EMG signal. 


\section{REFERENCES}

1. Kurek S, Rachwal T. Development of entrepreneurship in ageing populations of The European Union. Procedia Soc Behav Sci. 2011;19:397-405.

2. Carolino JA, Soares ML, Cândido GA. Envelhecimento e cidadania: possibilidades de convivência no mundo contemporâneo. Qualit@s Rev Eletrônica 2011;1(1):1-11.

3. Zacaron KAM, Dias JMD, Abreu NS, Dias RC. Nível de atividade, dor e edema e suas relações com a disfunção muscular do joelho de idosos com osteoartrite. Rev Bras Fisioter. 2006;10(3):279- 84.

4. Vasconcelos KSS, Dias JMD, Dias RCI. Relação entre intensidade de dor e capacidade funcional em indivíduos obesos com osteoartrite de joelho. Rev Bras Fisioter. 2006;10(2):213-8.

5. Santos MLAS, Gomes WF, Bárbara BZ, Rosa NMB, Pereira SP, Dias JMDD, et al. Desempenho muscular, dor, rigidez e funcionalidade de idosas com osteoartrite de joelho. Acta Ortop Bras. 2011;19(4):193-7.

6. Kendall FP, McCreary EK, Provance PG, Rod MM. Músculos-provas e funções com postura e dor. 5a ed. São Paulo: Manole; 2007.

7. Bracco D, Thiébaud D, Chioléro RL, Landry M, Burckhardt P, Schutz Y. Segmental body composition assessed by bioelectrical impedance analysis and DEXA in humans. J Appl Physiol (1985). 1996;81(6):2580-7.

8. Kyle UG, Genton L, Karsegard L, Slosman DO, Pichard C. Single prediction equation for bioelectrical impedance analysis in adults aged 20-94 years. Nutrition. 2001;17(3):248-53

9. Hermens HJ, Freriks B, Disselhorst-Klug C, Rau G. Development of recommendations for SEMG sensors and sensor placement procedures. J Electromyogr Kinesiol. 2000;10(5):361-74.

10. Fernandes MI. Tradução e validação do questionário de qualidade de vida específico para osteoartrose WOMAC (Western Ontario and McMaster Universities) para a língua portuguesa [dissertação]. São Paulo: Universidade Federal de São Paulo, Escola Paulista de Medicina; 2001.

11. Barbetta P. Estatística aplicada às ciências sociais. Florianópolis: Editora UFSC; 2006.

12. Rosner B. Fundamentals of Biostatistics. 7th ed. Massachusetts: Brooks/Cole; 2011.
13. Charro MA, Bacurau RFP, Navarro F, Pontes Jr FL. Manual de avaliação física. São Paulo: Phorte, 2010.

14. WHO - World Health Organization. Obesity - preventing and managing the global epidemic. World Health Organ Tech Rep Ser. 2000; 894:1-253.

15. Mainenti MR, Rodrigues Ede C, Oliveira JF, Ferreira Ade S, Dias CM, Silva AL. Adiposity and postural balance control: correlations between bioelectricalimpedance and stabilometric signals in elderly Brazilian women. Clinics (São Paulo). 2011;66(9):1513-8.

16. Deurenberg P, Andreoli A, Borg P, Kukkonen-Harjula K, de Lorenzo A, van Marken Lichtenbelt WD, et al. The validity of predicted body fat percentage from body mass index and from impedance in samples of five European populations. Eur J Clin Nutr. 2001;55(11):973-9.

17. Becker R, Berth A, Nehring M, Awiszus F. Neuromuscular quadriceps dysfunction prior to osteoarthritis of the knee. J Orthop Res. 2004;22(4):768-73.

18. Pap G, Machner A, Awiszus F. Strength and voluntary activation of the quadriceps femoris muscle at different severities of osteoarthritic knee joint damage. J Orthop Res. 2004;22(1):96-103.

19. Gonçalves M, Silva SRD. Análise de variáveis eletromiográficas durante contração isométrica fadigante. Salusvita. 2007;26(1):39-51.

20. Foley SJ, Lord SR, Srikanth V, Cooley H, Jones G. Falls risk is associated with pain and dysfunction but not radiographic osteoarthritis in older adults: Tasmanian Older Adult Cohort study. Osteoarthritis Cartilage. 2006;14(6):533-9

21. Silva SRD, Gonçalves M. Análise da fadiga muscular pela amplitude do sinal eletromiográfico. Rev Bras Ci Mov. 2003;11(3):15-20.

22. Orsatti FL, Dalanesi RC, Maestá N, Náhas EAP, Burini RC. Redução da força muscular está relacionada à perda muscular em mulheres acima de 40 anos. Rev Bras Cineantropom Desemp Hum. 2011;13(1):36-42.

23. Carmeli E, Imam B, Merrick J. The relationship of pre-sarcopenia (low muscle mass) and sarcopenia (loss of muscle strength) with functional decline in individuals with intellectual disability (ID). Arch Gerontol Geriatr. 2012;55(1):181-5. 